\title{
SLEEP PROBLEMS AND ADHD
}

The prevalence of sleep problems and their associations with quality of life (QOL), school attendance, and family impacts in children with ADHD were determined in a study at Royal Children's Hospital, University of Melbourne, Australia. Caregivers' (primarily mothers) in 239 eligible families reported sleep problems during the previous 4 weeks that were mild in $28.5 \%$, and moderate or severe in $44.8 \%$ of ADHD children. Moderate or severe sleep problems were associated with impaired child QOL, child daily functioning, caregiver mental health, and family functioning. Children with sleep problems were more likely to miss or be late for school, and their caregivers were more likely to be late for work. Caregivers for sleep deprived ADHD children were 2.7 times more likely to be clinically depressed or anxious. Pediatricians had enquired about child sleep problems in $45 \%$ cases, and caregivers had received treatment advice in $60 \%$ of those affected. (Sung V, Hiscock H, Sciberras E, Efron D. Sleep problems in children with attention-deficit/hyperactivity disorder. Arch Pediatr Adolesc Med April 2008;162:336-342). (Respond: Valerie Sung MBBS, Centre for Community Child Health, Royal Children's Hospital, Flemington Road, Parkville, Victoria, Australia 3052. E-mail: valeriesung(circh.org.au).

COMMENT. Sleep problems in schoolchildren with ADHD are common and are associated with impairment of QOL, daily functioning, and school attendance. Caregivers' mental health and work attendance are also adversely affected. Treatment of sleep disorders might improve daytime attention and behavior and lessen the need for stimulant medication.

Two further articles and an editorial on sleep disorders and behavior appear in the same issue of this journal. Hvolby A and associates in Denmark (Arch Pediatr Adolesc Med 2008;162:323-329) found that children with ADHD have significantly longer sleep onset latency and a more irregular sleep pattern than controls. Comorbid ODD is not related to sleep disorders. Parents over-report irregular sleep patterns as compared to objective measurements using actigraphy.

In a study at Brown University, Rhode Island Hospital, Providence, Owens JA and associates (Arch Pediatr Adolesc Med 2008;162:313-321) report risk factors, including overweight, short sleep duration, and comorbid sleep disorders, affecting behavioral outcome in children with sleep-disordered breathing (SDB). More than half (56\%) of the sample was overweight, and more than one-third (36\%) were short sleepers. Almost half $(49 \%)$ had an additional sleep diagnosis, particularly insomnia. SDB severity has a weaker influence on behavioral outcome. Behavior problems were reported in $47 \%$; ADHD in $23 \%$.

In the editorial, Cao M and Guilleminault C, Sleep Disorders Clinic, Stanford University, emphasize cultural differences in sleeping behaviors including cosleeping, limitations of polysomnography as a diagnostic tool, orthodontic problems as a cause of parasomnias, sleep-related disorders as cause or complication of $\mathrm{ADHD}$, and the relation of early childhood sleep disorders to adolescent and adult emotional and behavioral problems. The relation between sleep duration and childhood obesity requires more investigation, and the importance of stricter sleep schedules in young children needs more emphasis in parental education. (Arch Pediatr Adolesc Med 2008;162:385-389). 